\title{
The Leg Length Discrepancies: Clinical and Radiographic Criteria for Evaluation
}

\author{
Luigi Molfetta ${ }^{1 *}$, Andrea Casabella ${ }^{1}$, Augusto Palermo ${ }^{2}$, De Ponte Conti B ${ }^{3}$ \\ ${ }^{1}$ School of Medical and Pharmaceutical Sciences, Research Centre on Osteoporosis and Osteoarticular Diseases, University of Genoa, Italy \\ 2Department of Orthopedic, Italian Auxologic Institute, IRCCS Capitanio Milano, Milan, Italy \\ ${ }^{3}$ Degree course on Motory Science, D'Annunzio University of Chieti-Pescara, Italy
}

${ }^{\star}$ Correspondence to: Luigi Molfetta, School of Medical and Pharmaceutical Sciences, Research Centre on Osteoporosis and Osteoarticular Diseases (CROPO) University of Genoa, Genoa, Italy; E-mail: Imofetta@libero.it

Received: June 15, 2018; Accepted: June 23, 2018; Published: June 24, 2018;

\section{Summary}

The heterometry of the lower limbs in the developmental age can influence the development of the rachis, generating axial deviations or not good adaptations in walking; in adulthood it can have an important role in low back pain. In general, a heterometry must always be compensated in the evolutionary age, in order to align the rachis-pelvis-lower limb system for an optimal mechanical equilibrium of the subject. The clinical iter that leads to highlight and quantify the heterometry of the lower limbs is based on a careful observation of clinical and radiographic features; from the integration of all the references comes a reliable value for the purpose of mechanical compensation.

Keywords: lower limbs, heterometry, clinical examination

\section{Introduction}

In the global kinesiological assessment of a child is necessary a preliminary identification of a possible heterometry of the lower limbs (h.l.l.), considering the anatomical-functional relationship that exists between the rachis and the lower limbs for balance and correct joint function. The iliac bones are correlated in synergy with the lower limbs, the sacrum with the vertebral column [1]. In the developmental age the lower limbs are frequently with length differences often underestimated or more frequently misunderstood, with disharmonic reflexes on the growth or rehabilitative programming of the small patient. The h.l.1., can be idiopathic in the absence of diseases that cause anatomical districts and articular alterations or secondary to congenital or acquired joint pathologies of the hip (Epiphysiolysis, Dysplasias, Osteochondrosis, etc.), of the knee (asymmetric axial deviations) and of the foot (outcomes of diseases congenital, asymmetric pronator syndromes, etc.) [2].

The lack of or insufficient correction or often the overcorrection of the heterogeneous limb in the developmental age can exert an influence on the mechanical arrangement of the pelvis and of the rachis. There is often a discrepancy between specialists, about the presence and the extent of h.l.1. not carefully measured or based only on an inaccurate radiographic report. There is a clinical study, a semiological iter for the definition of h.l.1., supported by a radiographic study. A group of patients in developmental age has been evaluated by more specialists to identify h.l.1., and study the incidence of detection errors and their degree of significance (Figure 1).

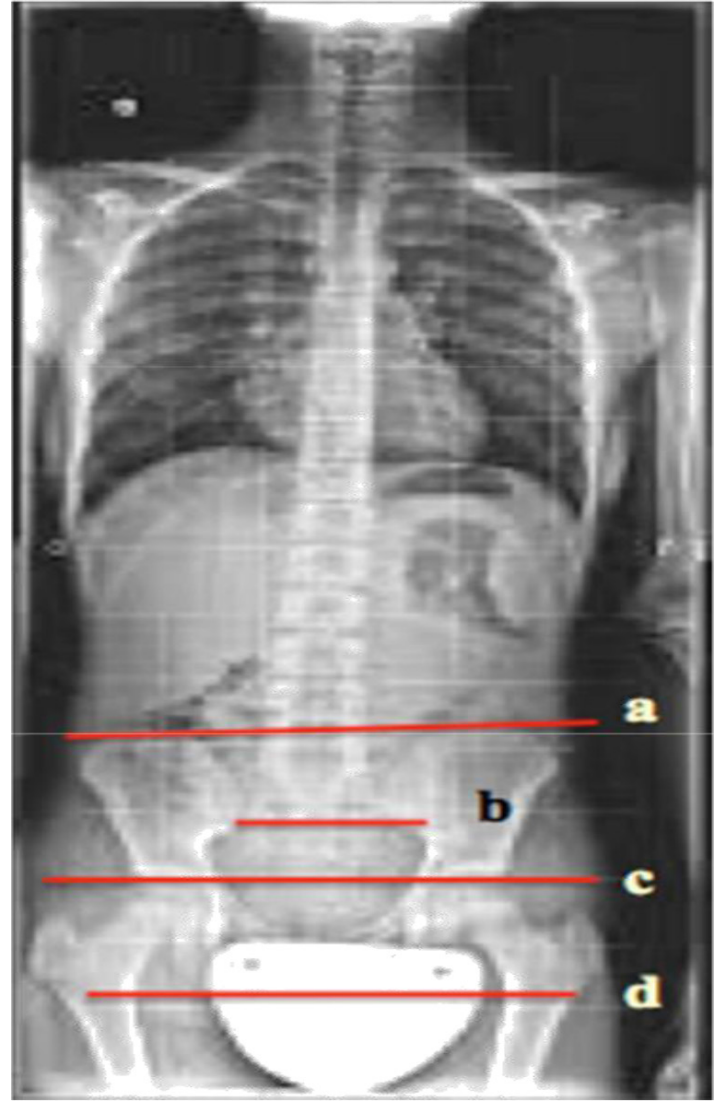

Figure 1: a: Iliac Crest Line; b: Sincondrosis Sacroiliac Line; c: Heads Femur Line; d: Little Trochanter Line 


\section{Material and Method}

\section{Clinical Analysis}

Evaluation of the patient with an h.l.l., must be done during walking, in orthostasis and in clinostasis, looking for repeatable reperiences for all the evaluators. They must be correlated with the radiographic data. The patient's walking allows detecting disharmony in the step or lameness; in general, a heterometry is manifested in the ambulation when it is equal to or greater than $1.0 \mathrm{~cm}$, if it is of an inferior value it finds an intrinsic compensation and cannot be documented in the passage [3].

Clinical observation in walking in a posterior view better demonstrates this, since the posterior and Superior Iliac Spines (SIPS) can be seen. In orthostasis barefoot, on a podoscope, the following are to be considered:

a. The level of Iliac Crests, assessed through the placement of the examiner's hands on the same. This assessment may be imprecise, related to the experience of the examiner and disturbed by the presence of fat, the tickling of the patient, etc.

b. The examination of the Postero-Superior Iliac Spine (SIPS), particularly evident in the thin subjects.

c. The alignment of the gluteal folds and the poplite folds.

d. Plantar support and asymmetric pronator syndromes, due to h.l.1.

In clinostasis after having mobilized the hips to eliminate contractures and bad functional adaptations and aligned the limbs slightly dividing, we proceeded to measure:

a. The medial spino-malleolar distance, with limbs in full extension, from the antero-superior iliac spine up to the apex of the medial malleolus, in comparison with the contralateral limb.

b. The medial navel-malleolar distance from the amelic to the apex of the medial malleolus.

c. The unevenness of the knees flexed, at $90^{\circ}$, with the feet juxtaposed and aligned on the back side of the heel; a repere on the knees records the difference in height of the rotule [3-7].

\section{Radiographic Study}

On the panoramic radiograph of the rachis in anterior-posterior projection the radiologist often reports the data of an heterometry, referring to the comparative level of the iliac crests projected on the radiographic grid. Considering the radiographic magnification (on average of $15 \%$ ) and the symmetry of the pelvis in the radiography, the reading of an X-ray is based on the observation of the following features:

a. The level of the iliac crests, projected on the radiographic grid, is the most widely used or even the only one.

b. The tangent to the femoral heads, a true reference for the metric variations of the limbs.

c. The tangent to sacro-iliac synchondrosis, inferiorly, considered a fixed, little variable and therefore reliable. d. The horizontal joining half of the small trochanters, if they appear symmetrical; this datum correlates with the tangent of the femoral heads (Figure 2).

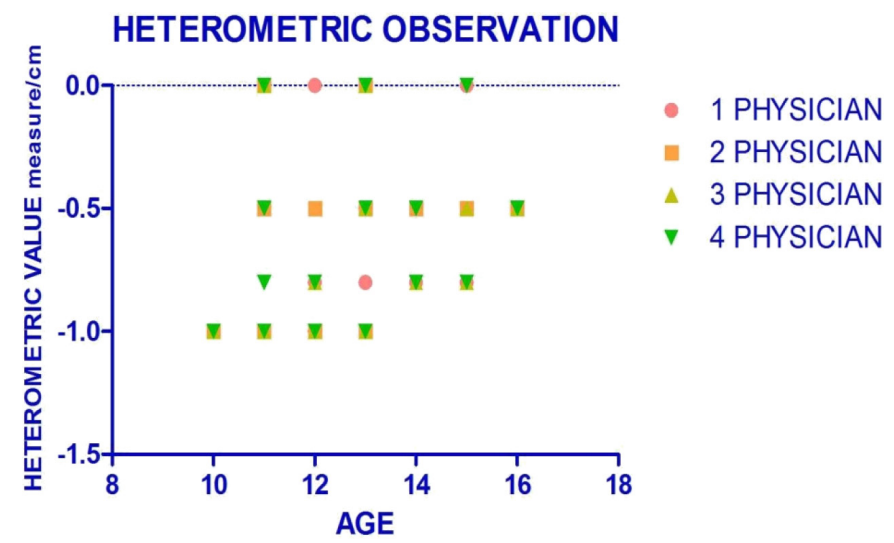

Figure 2: Heterometric values in study population.

\section{Case Material}

Twenty patients in the developmental age, 13 females and 7 males, aged between 10 and 16 years (average age of 12.8 years) who came to the observation for presumed scoliotic deficiency, were subjected to the examination of vertebral pathology, to clinical and radiographic evaluation for the recognition of an eventual h.l.l. Patients with pelvic dysmorphism and dysplastic hip disease were excluded. Each small patient was evaluated by 4 orthopaedic specialists (Table 1 ).

Table 1. Physician's Evaluation in Study Population.

\begin{tabular}{|c|c|c|c|c|c|c|}
\hline $\mathbf{N}^{\circ}$ & $\mathbf{s e x}$ & age & $\mathbf{1}^{\circ}$ Physician & $\mathbf{2}^{\circ}$ Physician & $\mathbf{3}^{\circ}$ Physician & $\mathbf{4}^{\circ}$ Physician \\
\hline 1 & $\mathrm{~F}$ & 12 & $-1 \mathrm{~cm}$ & $-1 \mathrm{~cm}$ & $-1 \mathrm{~cm}$ & $-1 \mathrm{~cm}$ \\
\hline 2 & F & 11 & $-0,5 \mathrm{~cm}$ & Non heter & Non heter & $-0,5 \mathrm{~cm}$ \\
\hline 3 & F & 14 & $-0,8 \mathrm{~cm}$ & $-0,5 \mathrm{~cm}$ & $-0,8 \mathrm{~cm}$ & $-0,8 \mathrm{~cm}$ \\
\hline 4 & M & 14 & $-0,5 \mathrm{~cm}$ & $-0,5 \mathrm{~cm}$ & $-0,8 \mathrm{~cm}$ & $-0,5 \mathrm{~cm}$ \\
\hline 5 & M & 12 & $-1 \mathrm{~cm}$ & $-1 \mathrm{~cm}$ & $-1 \mathrm{~cm}$ & $-1 \mathrm{~cm}$ \\
\hline 6 & F & 13 & No heter. & Non heter. & Non heter. & Non heter. \\
\hline 7 & F & 13 & $-0,8 \mathrm{~cm}$ & $-1 \mathrm{~cm}$ & $-1 \mathrm{~cm}$ & $-1 \mathrm{~cm}$ \\
\hline 8 & M & 15 & $-0,8 \mathrm{~cm}$ & $-0,5 \mathrm{~cm}$ & $-0,8 \mathrm{~cm}$ & $-0,8 \mathrm{~cm}$ \\
\hline 9 & F & 10 & $-1 \mathrm{~cm}$ & $-1 \mathrm{~cm}$ & $-1 \mathrm{~cm}$ & $-1 \mathrm{~cm}$ \\
\hline 10 & M & 12 & No heter & $-0,5 \mathrm{~cm}$ & $-0,8$ & $-1 \mathrm{~cm}$ \\
\hline 11 & F & 11 & $-0,5 \mathrm{~cm}$ & $-0,5 \mathrm{~cm}$ & No heter & $-0,8 \mathrm{~cm}$ \\
\hline 12 & F & 11 & No heter & $-0,5 \mathrm{~cm}$ & No heter & Non heter \\
\hline 13 & M & 15 & $-0,8 \mathrm{~cm}$ & $-0,5 \mathrm{~cm}$ & $-0,8 \mathrm{~cm}$ & $-0,8 \mathrm{~cm}$ \\
\hline 14 & F & 16 & $-0,5 \mathrm{~cm}$ & $-0,5 \mathrm{~cm}$ & $-0,5 \mathrm{~cm}$ & $-0,5 \mathrm{~cm}$ \\
\hline 15 & F & 14 & $-0,5 \mathrm{~cm}$ & $-0,5 \mathrm{~cm}$ & $-0,8 \mathrm{~cm}$ & $-0,5 \mathrm{~cm}$ \\
\hline 16 & F & 13 & No heter & No heter & $-0,5 \mathrm{~cm}$ & No heter \\
\hline 17 & M & 12 & $-0,8 \mathrm{~cm}$ & $-0,5 \mathrm{~cm}$ & $-1 \mathrm{~cm}$ & $-0,8 \mathrm{~cm}$ \\
\hline 18 & F & 13 & $-0,5 \mathrm{~cm}$ & $-0,5 \mathrm{~cm}$ & $-0,5 \mathrm{~cm}$ & $-0,5 \mathrm{~cm}$ \\
\hline 20 & M & 11 & $-1 \mathrm{~cm}$ & $-1 \mathrm{~cm}$ & $-1 \mathrm{~cm}$ & $-1 \mathrm{~cm}$ \\
\hline
\end{tabular}




\section{Results}

The overall data on the 20 patients analyzed by 4 specialists are summarized in Table 1. Each specialist had evaluated 7 clinical parameters and 4 radiographic parameters. There was a unanimous correspondence between the 4 specialists for 7 cases (35\%), a correspondence of 3 specialists on 4 for 10 cases (50\%), a correspondence of 2 specialists on 4 in 2 cases (10\%) and a complete discrepancy in 1 case (5\%) (Figure 2). Among the clinical parameters the Spine-malleolar distance and the evaluation in clinostasis with flexed knees were very important, which for cases with h.l.1. they are constant results for all observers. The other data have meant confirmation for the former. About the radiographic data, the most significant and constant datum was the repere of the femoral heads, compared to the reticulum of the iliac crests, considered the main reference in the radiographic reports. In the group of study patients, according to the 4 different specialists, higher mean heterometric values were observed for the observer $n^{\circ} 3$ and $n{ }^{\circ} 4$ with respect to the evaluation of the observer $n^{\circ} 1$ and $n^{\circ} 2$ (both with homogeneous heterometric values) resulting in a significant correlation of results, respectively: $-0.6400 \pm$ $0.3733 \mathrm{~cm} ;-0.6250 \pm 0.3726 ;-0.5500 \pm 0.3735 \mathrm{~cm} ;-0.5500 \pm 0.3204$ $\mathrm{cm}$. with $\mathrm{p}<0.0001$ (Figure 3 ). No significant correlation was found between age and the evaluations of the 4 observers (respectively; $\mathrm{p}=$ $0.40 ; \mathrm{p}=0.28 ; \mathrm{p}=0.68 ; \mathrm{p}=0.13=0.13$ ).

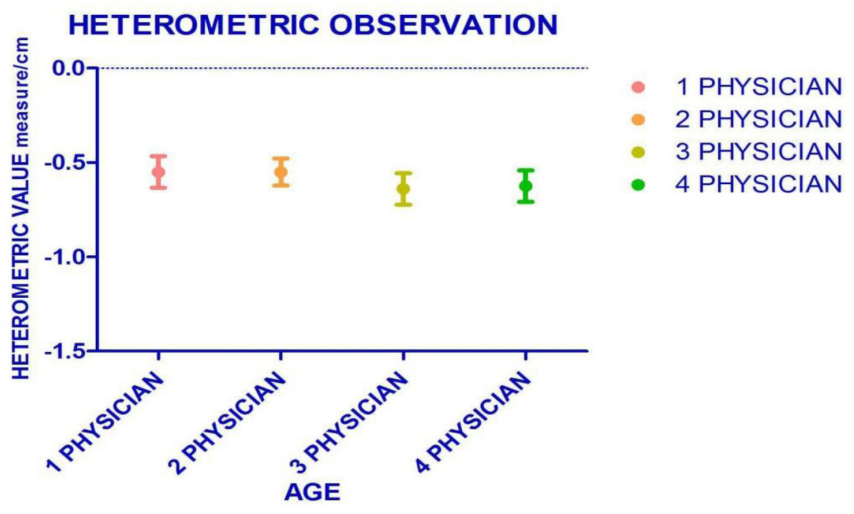

Figure 3: The mean resulting in heterometric evaluation: study population.

Therefore the search for an h.l.1. it does not correlate with the patient's age but with the accuracy of the clinical evaluation and with the observation of several semiological.

\section{Discussion}

An heterometry of the lower limbs causes a functional lumbar compensation curve; lumbar salience disappears both with the compensation of the same heterometry (equivalent rise) and with the Stagnara maneuver (deflection of the trunk in the discharge). The adaptive functional datum of the lumbar curve to the heterometry and its existence and existence. May be present in a patient with idiopathic scoliosis [4]. Preliminarily do not base the diagnosis of heterometry of the lower limbs on the sole radiographic reference, on presumed clockwise or anticorrosive rotations of the pelvis, without any anatomical-functional references. Some authors propose the use of QCT scans for data evaluation $[5,6]$. The evaluation starts from the clinical examination in its entirety and is answered in the radiographic data; the sum of the evaluations concludes the clinical reasoning on the subject and expresses the extent of any heterometry. The search for heterometry on the radiograph of the panoramic spine should not only limit references on iliac crest.

On the frontal plane it is possible to detect asymmetries of a hemi-pelvis on the sagittal plane with alteration of the anteroversion or retroversion and on the transverse plane for modification of the intra-and extra-rotation. Total in the presence of retroversion of an emi-pelvis, h.l.l. is only apparent thanks to an posture adaptation of homologous lower limb, sometimes inducing a sub-division of contralateral hemi-pelvis. In such cases, this is a question before considering dismetric limbs [7]. The success has been more than ever positive among the clinical signs some have the value of greater reliability as precision of the spino-malleolar distance or the evaluation of an inflection compared to others. Among the radiographic signs, the tangent to the femoral heads represents the main reference point associated with the other three parameters. For all physicians were observed a correlation of the data, with no relation between the age and the evaluations of the specialists. Respect the search for h.l.l. is correlates with the accuracy of the clinical evaluation and with the examination of several semiological parameters, integrated for a diagnosis of probable certainty $[8,9]$.

\section{References}

1. Morscher E (1972) Etiology and pathophysiology of leg length discrepancies. Orthopade 1: 1-8.

2. Papaioannou T, Stokes I, Kenwright J (1982) Scoliosis associated with limb-length inequality. J Bone Joint Surg Am 64: 59-62. [crossref]

3. Song KM, Halliday SE, Little DG (1997) The effect of limb-length discrepancy on gait. J Bone Joint Surg Am 79: 1690-1698. [crossref]

4. Leali Tranquilli P, Valassina A "Le dismetria degli arti", Argomenti di ortopedia e traumatologia Estratti, Verducci Editore.

5. GREEN WT, WYATT GM, ANDERSON M (1946) Orthoroentgenography as a method of measuring the bones of the lower extremities. J Bone Joint Surg Am 28: 60-65. [crossref]

6. Aaron A, Weinstein D, Thickman D, Eilert R (1992) Comparison of orthoroentgenography and computed tomography in the measurement of limblength discrepancy. J Bone Joint Surg Am 74: 897-902. [crossref]

7. Stanitski DF (1999) Limb-length inequality: assessment and treatment options. $J$ Am Acad Orthop Surg 7: 143-153. [crossref]

8. Goel A, Loudon J, Nazare A, Rondinelli R, Hassanein K (1997) Joint moments in minor limb length discrepancy: a pilot study. Am J Orthop (Belle Mead NJ) 26: 852-856. [crossref]

9. Shapiro F (1982) Developmental patterns in lower-extremity length discrepancies. $J$ Bone Joint Surg Am 64: 639-651. [crossref]

Citation:

Molfetta L, Casabella A, Palermo A, De Ponte Conti B (2018) The Leg Length Discrepancies: Clinical and Radiographic Criteria for Evaluation. Integr J Orthop Traumatol Volume 1(1): 1-3. DOI: 10.31038/IJOT.2018101 